\title{
Enhancing shopping experiences in smart retailing
}

\author{
Lorena Bourg $^{1} \cdot$ Thomas Chatzidimitris $^{2,8} \cdot$ Ioannis Chatzigiannakis ${ }^{3}$. Damianos Gavalas ${ }^{4,8}$ (D) \\ Kalliopi Giannakopoulou ${ }^{5,8} \cdot$ Vlasios Kasapakis $^{2,8}$. Charalampos Konstantopoulos ${ }^{6,8} \cdot$ Damianos Kypriadis $^{6,8}$. \\ Grammati Pantziou ${ }^{7,8} \cdot$ Christos Zaroliagis ${ }^{5,8}$
}

Received: 16 June 2020 / Accepted: 27 November 2020

(c) The Author(s), under exclusive licence to Springer-Verlag GmbH, DE part of Springer Nature 2021

\begin{abstract}
The retailing market has undergone a paradigm-shift in the last decades, departing from its traditional form of shopping in brick-and-mortar stores towards online shopping and the establishment of shopping malls. As a result, "small" independent retailers operating in urban environments have suffered a substantial reduction of their turnover. This situation could be presumably reversed if retailers were to establish business "alliances" targeting economies of scale and engage themselves in providing innovative digital services. The SMARTBUY ecosystem realizes the concept of a "distributed shopping mall", which allows retailers to join forces and unite in a large commercial coalition that generates added value for both retailers and customers. Along this line, the SMARTBUY ecosystem offers several novel features: (i) inventory management of centralized products and services, (ii) geo-located marketing of products and services, (iii) location-based search for products offered by neighboring retailers, and (iv) personalized recommendations for purchasing products derived by an innovative recommendation system. SMARTBUY materializes a blended retailing paradigm which combines the benefits of online shopping with the attractiveness of traditional shopping in brick-and-mortar stores. This article provides an overview of the main architectural components and functional aspects of the SMARTBUY ecosystem. Then, it reports the main findings derived from a 12 months-long pilot execution of SMARTBUY across four European cities and discusses the key technology acceptance factors when deploying alike business alliances.
\end{abstract}

Keywords e-Commerce $\cdot$ Retailer $\cdot$ Shopping mall $\cdot$ Inventory management $\cdot$ Ecosystem $\cdot$ Smart cities $\cdot$ Smart retailing . Geo-located marketing $\cdot$ Location-based search $\cdot$ Context awareness $\cdot$ Recommendation

Damianos Gavalas

dgavalas@aegean.gr

Lorena Bourg

lbourg@gmail.com

Thomas Chatzidimitris

tchatz@aegean.gr

Ioannis Chatzigiannakis

ichatz@diag.uniroma1.it

Kalliopi Giannakopoulou gianakok@ceid.upatras.gr

Vlasios Kasapakis

v.kasapakis@aegean.gr

Charalampos Konstantopoulos

konstant@unipi.gr

Damianos Kypriadis

dkypriad@unipi.gr

Grammati Pantziou pantziou@uniwa.gr
Christos Zaroliagis

zaro@ceid.upatras.gr

1 Planet Media Studios, Madrid, Spain

2 Department of Cultural Technology and Communication, University of the Aegean, Mytilene, Greece

3 Department of Computer, Control and Informatics Engineering, Sapienza University of Rome, Rome, Italy

4 Department of Product and Systems Design Engineering, University of the Aegean, Syros, Greece

5 Department of Computer Engineering and Informatics, University of Patras, Patras, Greece

6 Department of Informatics, University of Piraeus, Piraeus, Greece

7 Department of Informatics and Computer Engineering, University of West Attica, Athens, Greece

8 Computer Technology Institute and Press (CTI), Patras, Greece 


\section{Introduction}

The decline of isolated retailers and the rise of shopping malls have been major trends in retailing for decades, worldwide (Teller 2008). Retail agglomeration ${ }^{1}$ in shopping malls has been found useful to both consumers as well as firms. Shopping mall agglomeration offers the benefits of higher footfalls, shared-physical and digital-infrastructure, cooperative marketing plans and high impulse purchases (Sanyal and Ghosh 2017).

The fast-paced development of ICTs has also dramatically changed the way people shop in the past 15 years. Besides shopping at physical stores, with the aid of ICT, consumers are able to shop via the Internet. This new type of shopping mode (e-shopping or online shopping) frees consumers from having to physically visit stores and puts extra competitive pressure to small local retailers since customers often opt to shop online due to the attained cost and travel time savings (Hsiao 2009; Pantano and Timmermans 2014).

The aforementioned trends can be presumably reversed if retailers integrate ICT services in order to complement their traditional offline experiences with matching online offers; establish business "alliances" targeting economies of scale; engage themselves in providing innovative digital services.

The SMARTBUY ecosystem ${ }^{2}$ realizes the concept of a "distributed shopping mall", which allows retailers to join forces and unite in a large commercial coalition that generates added value for both retailers and customers. Along this line, the SMARTBUY ecosystem offers several new features: (i) inventory management of centralized products and services, (ii) geo-located marketing of products and services, (iii) location-based search for products offered by neighboring retailers, and (iv) personalized recommendations for purchasing products based on innovative recommendation systems.

For customers, the SMARTBUY approach combines the best of the two worlds: on the one hand, the benefits of online shopping (comparison of product prices across several stores, guarantee the availability of searched products, etc.); on the other hand, the diversified attractiveness of traditional store shopping (social interaction, entertainment, movement, trip chaining), live in-store information, offline retailer loyalty, low product performance risk, zero delay for product delivery, etc. (Gensler et al. 2017; Mokhtarian 2004).
Most recent approaches in smart retailing focus on the effective use of ubiquitous computing technologies to enable consumer profiling (Lin and Hong 2008) in order to provide intelligent in-store multimodal e-services (Pantano et al. 2017) and offer context-aware offers and promotions (Yang et al. 2008). In contrast, the emphasis of our work is on implementing the new business model of the distributed mall which combines the provisioning of innovative e-services to both retailers and customers with the motivation of physical visits of customers to retailer stores. This article provides an overview of the main architectural elements and innovative aspects of the SMARTBUY ecosystem. Moreover, it reports on a feasibility study for the introduced business model and discusses the experiences gained from the large-scale pilot execution of SMARTBUY in four European cities with diverse scale and retail market characteristics. Last, the paper investigates the factors influencing public acceptance of smart retail alliances.

It is noted that a preliminary version of this work appears in a conference paper published by Bourg et al. (2019). In comparison with (Bourg et al. 2019), this article presents the background concepts that motivated the development of the SMARTBUY ecosystem (see Sect. 3). We also provide an overview of the co-creation concept that has been adopted by SMARTBUY as well as the integration of the SMARTBUY ecosystem with the Organicity IoT platform ${ }^{3}$ (see Sect. 5.2). Finally, we provide a critical outlook of the results derived from the pilot execution of the SMARTBUY ecosystem in four European cities (see Sect. 6).

The remainder of the article is structured as follows. A succinct review of research related to the current work is presented in Sect. 2. Background information on the research motivation behind SMARTBUY is provided in Sect. 3. The scope of the SMARTBUY ecosystem as well as its main scientific, technical and business objectives are discussed in Sect. 4. An overview of the architecture of the SMARTBUY ecosystem, along with a description of the functionality and interoperation among its key modules, as well as the integration of SMARTBUY with an IoT platform is provided in Sect. 5. Results yield during the pilot tests of the SMARTBUY ecosystem across four European cities with different characteristics are presented and analyzed in Sect. 6. Conclusions drawn from this work along with directions for future research are offered in Sect. 7.

\footnotetext{
${ }^{1}$ Retail agglomeration is defined as the presence of a group of retail stores in close proximity, which cooperate and compete with each other simultaneously (Pantano and Timmermans 2014).

${ }^{2}$ Funded by the EC H2020 SMARTBUY project (2016-2019); http://smartbuy.tech/.
}

\footnotetext{
3 http://organicity.eu
} 


\section{Related research}

The six most-common indicators of smart cities are smart economy, smart people, smart governance, smart mobility, smart environment, and smart living (Bousios et al. 2017; Gavalas et al. 2017; Giffinger et al. 2007). Smart economy refers to technologies supporting new forms of collaboration and value creation that lead to innovation, entrepreneurship and competitiveness (Gretzel 2015; Kumar and Dahiya 2017). In this context, Pantano and Timmermans (2014) in their seminal work on smart retailing, emphasize that "the emerging idea of smart retailing would reflect a particular idea of retailing, where firms and consumers use technology to reinvent and reinforce their role in the new service economy, by improving the quality of their shopping experiences".

The smart retailing culture is characterized by the extensive use of mobile technologies, high connectivity, ubiquitous computing and contactless technologies, which enable consumers to experience shopping differently (Pantano and Priporas 2016). Thus, it is not surprising that there is an increasing awareness in marketing about the need to develop new mobile retailing strategies. Initial efforts involved the usage of SMS for advertising purposes (Amirkhanpour et al. 2014). Later, it expanded to mobile apps, which allow consumers to easily find, compare and order products, access news on products and services, create shopping lists, locate products and stores, etc. (Pantano and Priporas 2016). More recently, it encompassed smart stores with iBeacon infrastructures used to track the exact customer location and push product-related notifications, as well as NFC-equipped smartphones executing augmented reality apps to add virtual elements (textual information, images, videos, 3D animation clips, etc.) to products of interest when visiting the actual store (Pantano et al. 2017).

Among other aspects, knowledge management has been recognized as a critical success factor for (mobile) smart retailing, since data collection and consumer analysis can be facilitated by the adoption of new technologies (Lin and Hong 2008). Mobile/ubiquitous computing provides the required technological infrastructure to collect valuable knowledge from/for consumers (e.g., by codifying habits, processing clients' transaction information and identifying changes in consumers' behavior for supporting retailers, etc.), and feed knowledge into the service (i.e., by providing enriched information about certain products, or drawing the attention of consumers to -otherwise missedproducts of interest) Amaxilatis et al. 2018; Chatzigiannakis et al. 2011; Theodoridis et al. 2013). Along this line, mobile recommendation systems (RS) enable the adaptation of product information to make it more relevant to consumers' information needs (Gavalas and Kenteris 2011; Gavalas et al. 2013; Sassi et al. 2017). Mobile RSs may serve both in-store and online purchase situations as they "elicit the interests or preferences of individual users for products either explicitly or implicitly, and make recommendations accordingly" (Kowatsch and Maass 2010).

For instance, Yang et al. proposed a location-aware RS that correlates customers' shopping needs with locationdependent vendor offers and promotions (Yang et al. 2008). Yuan and Tsao (2003) introduced a framework which enables the creation of tailor-made campaigns targeting users according to their location, needs and devices' profile (i.e. contextualized mobile advertising). More recently, Chen and Ji (2016) proposed an approach for smart advertising in smart cities, with the use of crowdsourced trails provided by mobile and wearable IoT devices. The authors argue that, as user behavior is tracked via a device, analysis of these trails generates important clues for market planning.

SMARTBUY is grounded upon these developments in mobile retailing proposing the smart usage of technologies to enable changes in organizational processes, improve retail services and develop innovation management strategies. The SMARTBUY ecosystem serves a new collaborative and interactive shopping scenario, where consumers participate in the creation of the final service through their requests and interaction with provided technologies, and the system responds with personalized information and added-value services. Hence, the use of technology in SMARTBUY becomes smart by involving retailers and clients in a sort of "smart-partnership". This approach provides the basis for value creation via the implementation of smart marketing and e-commerce services which will be analyzed in the rest of this article.

Our research advances the state of the art in the field of smart retailing both from a business and a technical point of view. From a business perspective, we introduce the idea of the distributed mall. In contrast to (a) typical $\mathrm{e} / \mathrm{m}$-commerce services enabling online purchases over a centralized directories of products and, (b) technologyenriched physical retailer stores, our proposed business model enables the establishment of retail alliances providing added-value e-services while motivating physical visits of customers to retailer stores. From a technical perspective, our research makes important contributions to the fields of: geo-located marketing through tracking the behavior of anonymous consumers and improving the efficiency of marketing campaigns (see Sect. 5.1.2); context-aware recommendations engines through considering the location history of customers (among other contextual factors) to deliver more accurate product recommendations (see Sect. 5.1.4). 


\section{The SMARTBUY background}

Showrooming refers to the situation when a shopper visits a store to check out a product but then purchases the product online from home. This occurs because, while many people still prefer seeing and touching the merchandise they buy, many items are available at lower prices through online vendors. As such, local stores essentially become showrooms for online shoppers (Arora et al. 2017).

In the past few years, as online shopping exploded and smartphones became the norm, the showrooming phenomenon seemed to highly threaten store-based retailers. Online retailing still lags behind 'traditional' retailing in Europe, nevertheless, its market share steadily increases. According to a recent forecast analysis published by the Centre for Retail Research, ${ }^{4}$ the total store numbers in UK will fall by $20 \%$ and the share of online retail sales will increase by $21.5 \%$ by the end of the decade.

But now a recent report from BI Intelligence ${ }^{5}$ finds that retailers have discovered "reverse showrooming" or "webrooming", which is when consumers go online to research products, but then head to a brick-and-mortar store to complete their purchase. Reverse showrooming is actually nothing new. Since the early days of online shopping, more people have researched their shopping online than have actually bought there. What has changed is that retailers have begun to identify the reverse showrooming trend and the opportunity it offers to them, and they are now working to actively capture those sales.

Reverse showrooming is actually more common than showrooming. In the US, $69 \%$ of people reverse showroom, while $46 \%$ showroom, according to a Harris poll. ${ }^{6}$ Amazon remains the No. 1 place where showroomers end up making their purchases, but it constitutes an even more popular destination for reverse showroomers who ultimately buy elsewhere. Social media has also become a major referral source for brick-and-mortar chains, not just e-commerce sites.

But only recently have traditional retailers begun to capitalize on reverse showrooming. Offline retailers have realized they have a lot to offer, as long as they can integrate offline and digital experiences and beat e-commerce competitors on convenience. They use tactics like knowledgeable sales staff, in-store pick-up of online orders, in-store Wi-Fi,

\footnotetext{
$\overline{{ }^{4} \text { https://www.retailresearch.org/. }}$

5 https://www.businessinsider.com/reverse-showrooming-bricks-andmortar-retailers-fight-back-2-2014-2.

${ }^{6}$ https://theharrispoll.com/if-showrooming-was-a-holiday-trend-towatch-in-2012-then-webrooming-wherein-consumers-do-their-resea rch-online-and-then-head-to-the-store-to-actually-make-a-purchaseis-one-to-watch-in-2013-accord/.
}

and smartphone discounts that nudge showroomers to buy in-store (Mehra et al. 2017).

The retailers getting more benefit from this trend are the big ones. Big stores can offer an online and an optimized offline buying experience as well. They can offer in-store pick-up of online orders, in-store Wi-Fi and an online sales infrastructure. They can be present at big commercial areas, malls and they can rent big buildings for optimizing the purchasing experience.

Small retailers are in risk of losing a big opportunity with the rising of in-store sales triggered by the rising of the webroomer trend. A Forrester study showed that webrooming would result in $\$ 1.8$ trillion in sales by 2017 , versus $\$ 1.2$ trillion in 2012. ${ }^{7}$ In comparison, all e-commerce sales should reach $\$ 370$ billion in 2017.

Small and medium-sized retailers must find their way to become the store were the webroomers finally go to end the purchase process. They have the advantage of being able to provide their customers with convenient and local access to stores. What if small-to-medium sized retailers could be able to take advantage of their local position at the same time that they offer an integrated web/show room experience? What if they could jointly create and maintain an integrated digital catalogue of products and at the same time become the physical stores where to get them?

\section{SMARTBUY scope and objectives}

Small and medium-sized independent retailers, typically (and henceforth) referred to as SMEs (Small and Medium Enterprises) lack visibility in the digital world. A large percentage of small businesses in Europe do not have a website; only a few have their product catalogues on-line, while most of them rely on social media marketing to disseminate promotional offers. They are rarely visible on large commercial platforms. In addition, the use of commercial platforms is very costly for SMEs. On the other hand, many customers would be interested in supporting their local retail SMEs and buying from them instead of from popular e-commerce platforms. However, this is not straightforward in the absence of on-line platforms specifically dedicated to SMEs.

For small retailers who wish exposure of their products online, it would make sense to cooperate together at local level and create a local on-line catalogue of products where they could advertise to local consumers. Individual SMEs may lack necessary IT skills and visibility online, but if such a platform was created it would benefit all the small retailers involved. Nowadays, retail SMEs should obtain help to

\footnotetext{
7 https://www.capillarytech.com/blog/omnichannel/showroomin g-and-webrooming/.
} 


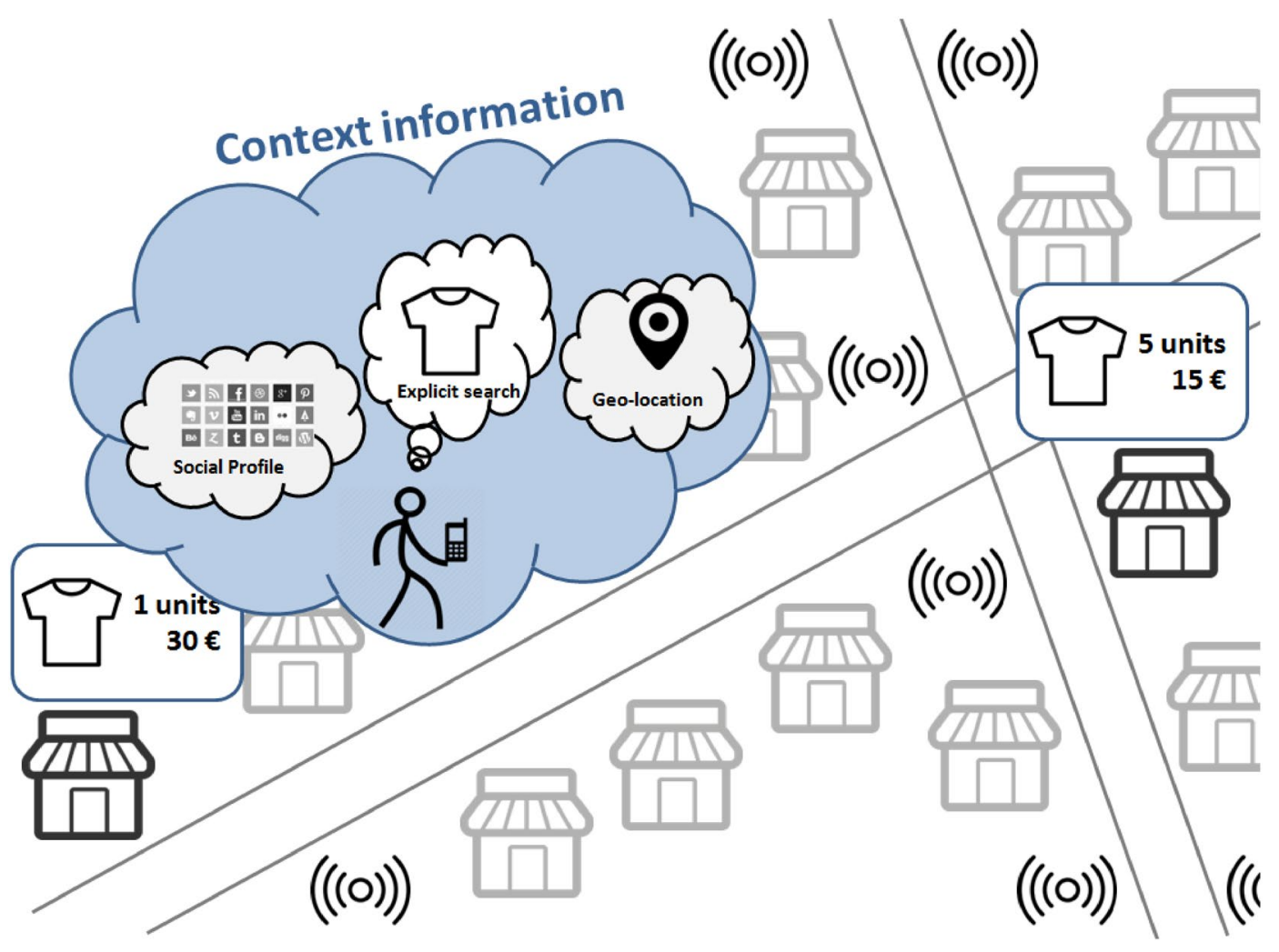

Fig. 1 Real-time centralized search for products offered by SME retailers' stores

create such platforms at the local level. In particular, assistance linked to IT skills and marketing would be necessary.

SMARTBUY contributes in closing this gap providing the technological infrastructure for small and medium sized retailers to become the place where they can easily make their products and promotions visible on-line for local audiences at the right moment; just in their smartphone and providing them also with competitive advantages of e-commerce: comparison of prices, choice of providers, reviews and specification awareness, guarantee of availability, etc. SMARTBUY provides SMEs retailers with an integrated suite of services allowing them to apply the tactics typically practiced by big retailers, thus offering an integrated digital and physical buying experience to customers.

SMARTBUY is thought as a service that achieves its bigger potential when applied in a delimited geographical area with dense deployment of commercial stores such as a city center. The service provides a location-based e-commerce ( $l$-commerce) integrated infrastructure for all the small retailers in a geographical area to use. The $l$-commerce infrastructure allows conducting centralized searches of products provided by the stores. Customers can have access to realtime information of the products, prices, availability, etc., with the convenience of being able to physical purchase in a local, near-to-home store, thus saving costs and delivery delays due to shipment.
SMARTBUY turns the physical stores of smart cities to a smart geographically distributed mall by providing the logical consistency needed for conducting centralized searches in heterogeneous and geographically distributed physical stores. Figure 1 shows how potential customers with their smartphones are able, using the SMARTBUY ecosystem, to receive real time information with respect to the availability of the products that they wish to purchase from nearby stores. This awareness can be achieved through an explicit search on the SMARTBUY's retailer's stores databases but also through available location-based services (see Sect. 5.1.2).

The SMARTBUY services are based on mature communication and information infrastructure components provided by the partners of the consortium. Nevertheless, a large-scale demonstration of the service is needed in order to validate the hypothesis on the impact that the service may have on the increase of sales and competitiveness of the small retailers.

The SMARTBUY ecosystem pursues the following main scientific, technical and business objectives:

- Provision of services and functionalities targeted to two different user types, SME retailers and potential customers.

- Services provision to potential customers allowing to: 


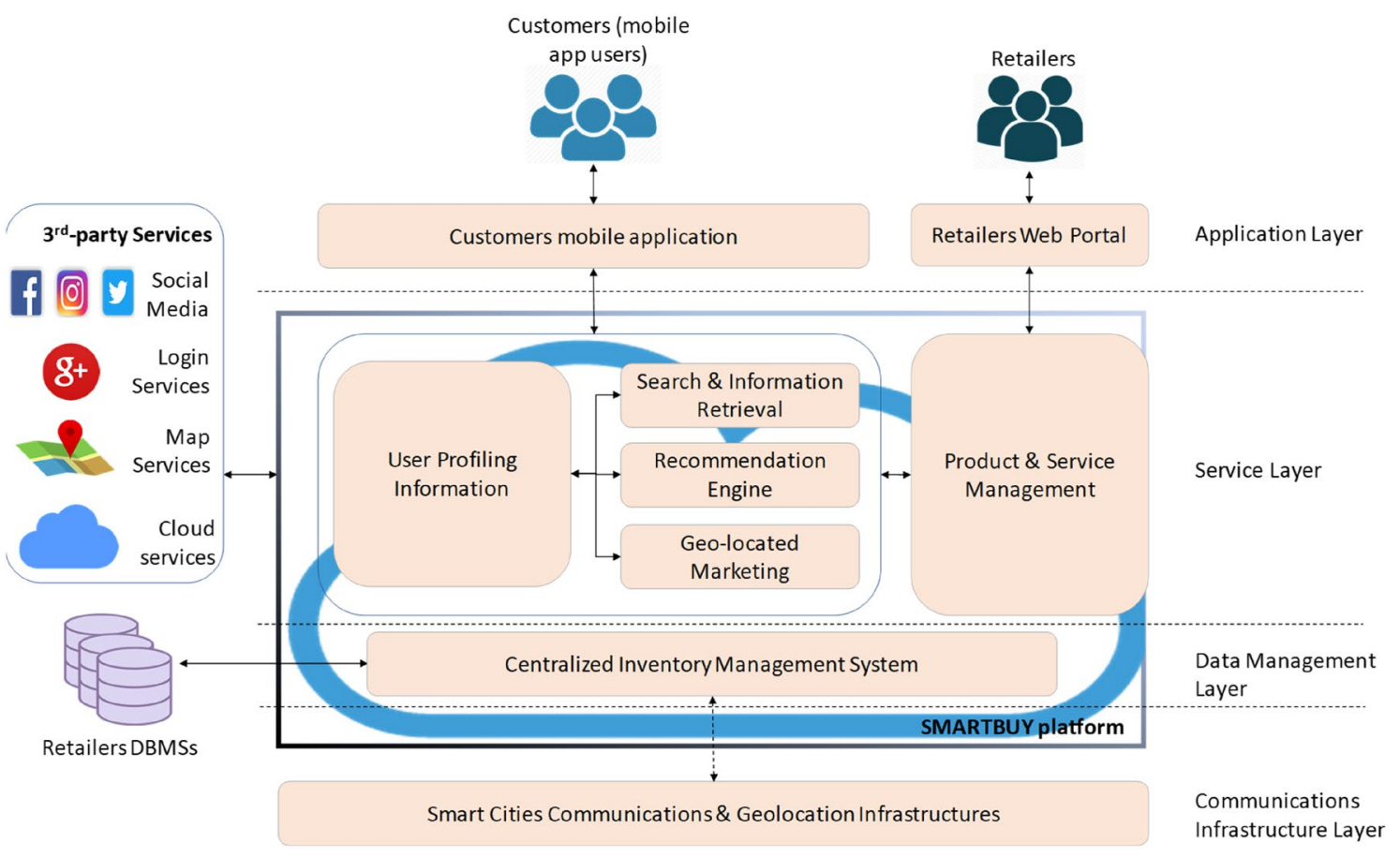

Fig. 2 The architecture of the SMARTBUY ecosystem

o Conduct geo-located, centralized and ubiquitous searches on the inventory of services and products of SMARTBUY member-stores.

o Check the availability, characteristics, price and reviews by third-parties of the products and services of their interest.

o Reserve the selected products and/or services online if the store had previously enabled this option.

o Review and share the acquired product and/or service online.

o Receive personalized recommendations and offers about products and services sold on nearby stores based on several contextual factors.

- Services provision to retail SMEs stores allowing to:

o Integrate their services and products databases to an integrated geo-localized sales ecosystem, attracting local audiences and increasing sales.

o Keep track of their inventory online.

o Enable online visibility, publication of their catalogue of products, and sales at low cost.

o Offer personalized and geo-located information and promotions to potential customers.

o Provide customized information about their products and services

o Provide real-time information about the availability of their products and services.
- Provision of a customized multichannel system for the interaction of potential customers with the SMARTBUY ecosystem (web portal and mobile app which takes advantage of geolocation capabilities).

- Large scale pilot experimentation and validation of the SMARTBUY ecosystem (especially wrt sales and profit increase as well as the ability to reach new potential customers) in several urban environments with diverse geographical, cultural and market characteristics.

\section{Overview of the SMARTBUY architecture and services}

The high-level architecture of the SMARTBUY ecosystem is illustrated in Fig. 2. The ecosystem consists of four layers: the communications infrastructure layer, the data management layer, the services layer, and the applications layer.

The SMARTBUY ecosystem is built upon a cloud infrastructure platform (communications infrastructure layer), fulfilling requirements of security and privacy, scalability and interoperability on top of which several modules have been deployed. The SME's database management systems can be integrated through a centralized inventory management system that comprises the data management layer.

The new features of the SMARTBUY ecosystem are materialized through four core services which (along with the user profiling module) comprise the services layer: 
product \& service management, geo-located marketing, search \& information retrieval, and the recommendation engine. Upon the services layer, several applications can be developed.

The modular architecture of the SMARTBUY ecosystem allows its integration with IoT platforms in order to use their tools for application development towards large-scale demonstrations and experimentations in smart cities, engaging not only IT specialists but also the whole range of smart city stakeholders.

In the remainder of this section, we shall present the four core SMARTBUY services as well as the integration of the SMARTBUY ecosystem with the IoT Organicity platform.

\subsection{The SMARTBUY core services}

In this section, we describe the different core services integrated in the SMARTBUY ecosystem, emphasizing on their innovative aspects in relation to the state of the art.

\subsubsection{Product and services data management}

The main interface among the retailers and the SMARTBUY ecosystem is the retailers' web portal (see Fig. 3). The latter is a web interface which offers the following key functionalities (SMARTBUY 2017b):

- User management: registration and authentication of users (store managers).

- Creation/editing of store (retail) details, like title, location, description, contacts, opening hours, etc.

- Creation/editing of catalogues.

- Creation/editing of products: name, category/subcategory, attributes (e.g. color, weight, size), short/long description, price, stock, pictures.

- Product promotions management: product, description, discount, start/end activation date.

- Statistics, like most viewed products, origin (location) of visitors, peak hours in the area of the store.

The retailers' portal adopts responsive design principles; hence, it may be accessed from PC, tablet or smartphone.

It is noted that SMARTBUY does not offer any web interface to customers to lookup store and product details, as it is intentionally oriented towards mobile users. However, in order to maintain a degree of exposure and visibility in the web, the ecosystem encompasses a tool which enables the indexability all products created under the retailers portal by search engines (it generates a 'webpage' for each product with automatically created metatags and alternative text for images); hence, it allows web users to search for SMARTBUY products online and view their key properties.
Clearly, the variety and the multitude of retailers participating in SMARTBUY as well as the variety and quantity of their uploaded products and services becomes a critical factor for the sustainability of the SMARTBUY ecosystem, because it escalates the options that potential buyers have for finding products and services of interest, hence, it improves the appeal of the ecosystem as a whole. The growth of the SMARTBUY community, in turn, allows retailers to exploit innovative 1-commerce services at the expense of a reasonable cost.

\subsubsection{Geo-located marketing}

Due to the ubiquity of mobile devices, the collection of large-scale, longitudinal data about human mobility is now commonplace. Medium-to-high resolution mobility data of individuals and signals of entire social systems can be captured from systems designed to enable communication and connectivity, such as cellular networks or Wi-Fi APs (access points) (Sapiezynski et al. 2015). Understanding and modeling the way humans move in urban contexts is useful to many applications, like vehicle traffic forecasting, human queues and crowd estimation, detection of human encounters, intelligent personal assistants (Meneses and Moreira 2012).

Mobility traces are highly unique and identify individuals with substantial accuracy. Sensitive features can be extracted from mobility data, including home and work locations, visited places, or personality traits (Sapiezynski et al. 2015).

In the context of SMARTBUY, we hypothesize that human mobility data inferred from Wi-Fi APs could boost the efficacy of marketing campaigns pursued by individual retailer-members or by the entire system as a whole (SMARTBUY 2017a). The exploitation of the SMARTBUY geo-marketing tools requires that the APs of the retailermembers are configured so as to connect to the CityPassenger's CityScope gateway platform. ${ }^{8}$ The motivation behind using CityScope is that small retailers cannot handle the cost of hiring a full-time network administrator. With CityScope, management tasks are simplified and integrated into a unique tool that provides multiple functionalities: data analytics, management of connected devices, security, control, information exchange between remote APs, etc.

The key idea behind SMARTBUY's geo-marketing is to use the Wi-Fi APs installed on retailers' premises as customer proximity detectors. The APs are able to capture the presence of users whose devices can be either registered or unregistered in the wireless network. The former connect to the AP (either automatically or manually through the SMARTBUY-branded Wi-Fi captive portal,

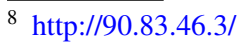




\section{smartbuy / your products $\quad$ Choose Language - Marc000 .}

* Your Retails , Tienda Talavera , Catalog IT Equipment , Products s Update Product

\section{UPDATE YOUR PRODUCT}

Product Data
Product Name
Portatil Asus | 400GB| 16GB |14" |i5
Price
1200

Stock Number

Description

ASUS presenta el nuevo UX30, un portátil muy fino y ligero a la vanguardia en diseño y tecnología. Con un procesador Intele Core ${ }^{\mathrm{r} w} 2$ Due el UX30 exhibe Product Detail

ASUS presenta el nuevo UX30, un portátil muy fino y ligero a la vanguardia en diseño y tecnología. Con un procesador Intele $\mathrm{Core}^{\mathrm{rw}} 2 \mathrm{Duo}$ el UX30 exhibe
Product Categories

Categories

$\checkmark$ Video

- Music

Books \& Audible

Movies, TV, Music \& Games

Electronics \& Computers

Home, Garden, Pets \& DIY

Toys, Children \& Baby

口Toys \& Games

口Baby

口Kid's Clothing

QBaby Wish List

口Family

Clothes, Shoes \& Jewelry

Car \& Motorbike

Business, Industry \& Science

Handmade

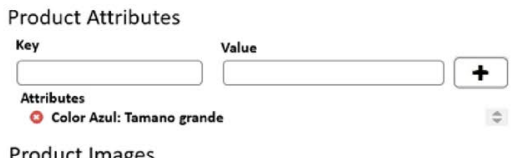

Product Images

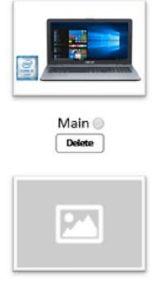

Main

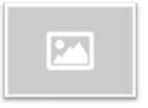

Main

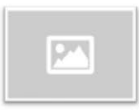

\begin{tabular}{|l} 
Main \\
Dosete
\end{tabular}

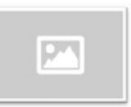

Main

(a)

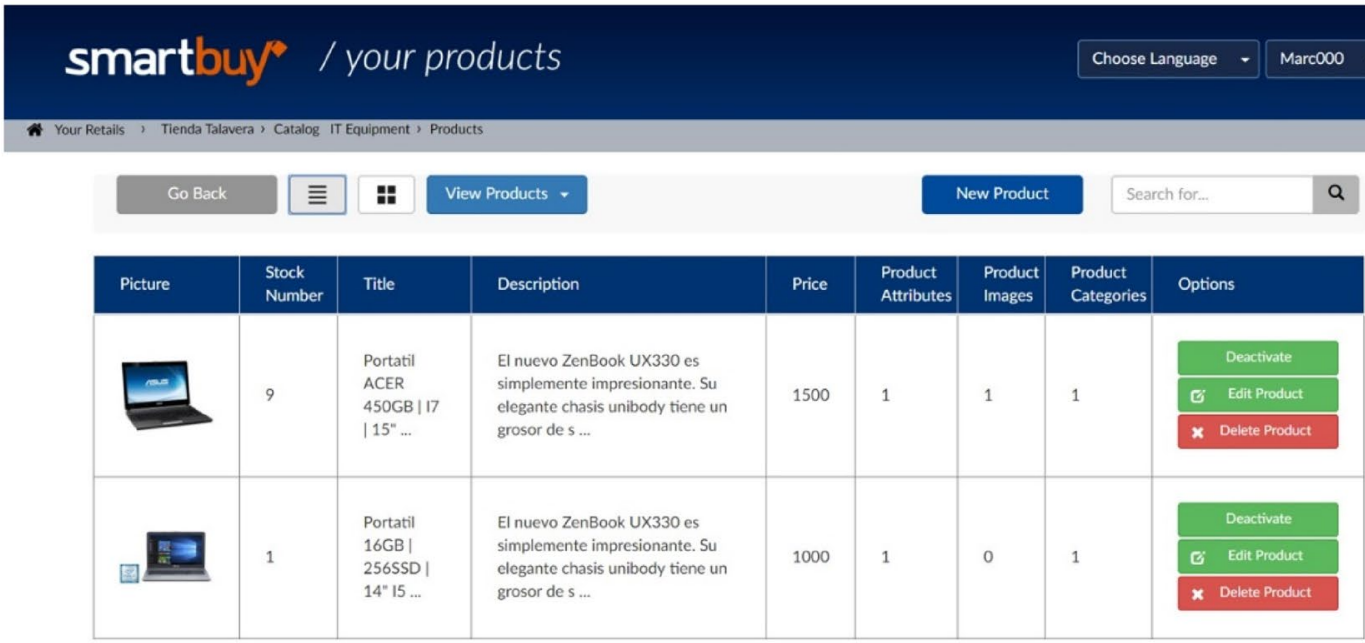

(b)

Fig. 3 SMARTBUY retailers web portal: new product upload and inventory management

as shown in Fig. 4a (it is noted that the captive portal can be exploited to promote the SMARTBUY brand to nonregistered consumers and encourage them to discover the services offered). The latter are detected through the probe request frames which are periodically transmitted from the $\mathrm{Wi}$-Fi radios of their smartphones (the unique MAC address of the radio transceiver is indicated on the header of those frames and may be used to identify users). The retailers APs are configured so as to report captured $\mathrm{Wi}-\mathrm{Fi}$ signals to CityScope. Among others, CityScope enables the information exchange among SMARTBUY-branded APs, even if they belong to different LANs and different retailers, effectively forming a wireless geo-marketing APs cluster at a city level. It is noted that the methods used for 
Fig. 4 SMARTBUY geomarketing tool: a SMARTBUYbranded WiFi captive portal; b Area coverage of retailersowned WiFi APs

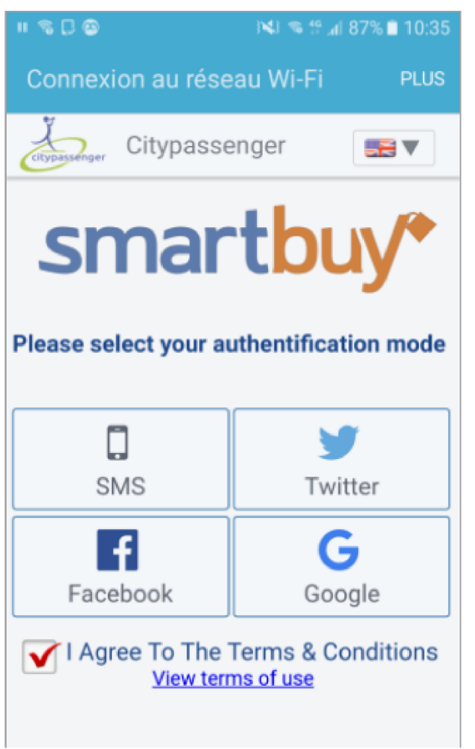

(a)

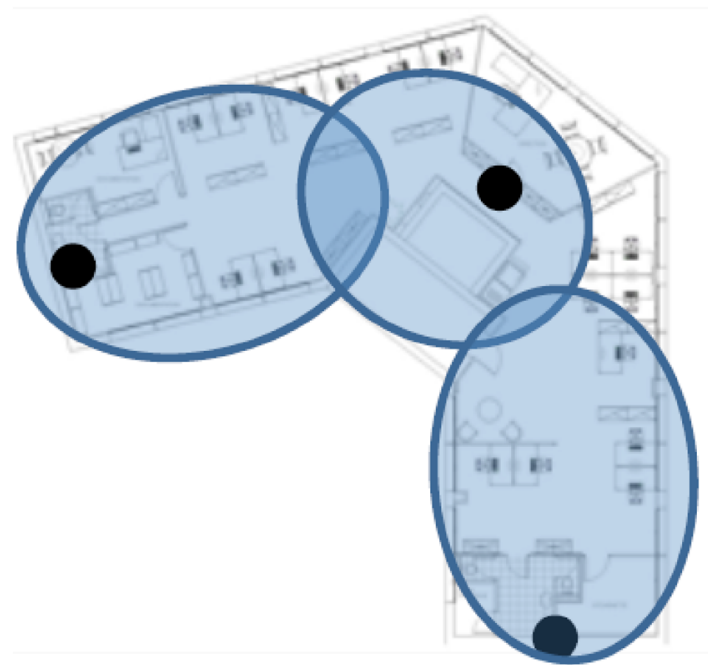

(b) the collection, anonymization, and storage of data comply with GDPR.

This city-scale customer mobility inference infrastructure offers numerous advantages to the retailers. The first one is to be informed when a registered consumer is around, not only in the vicinity of their own store but also when connected to another SMARTUBY hotspot in the broader area (see Fig. 4b, which illustrates the area covered by APs which are all connected to CityScope gateway to enable the seamless monitoring of customers' trajectory). The user may then be sent SMS notifications for personalized offers and discounts directly to her smartphone or be invited to install the mobile application and engage with the SMARTBUY community. The second one is to yield statistics about the connection of anonymous devices (i.e. not registered in SMARTBUY) based on the logs systematically recorded by APs. These statistics are important to gain insights about the evolution of physical stores' human traffic. At a higher level, these data can be used to track the behavior of anonymous consumers (trajectories, time spent at each location, etc.) in an area with several deployed Wi-Fi APs. User mobility data captured by the geo-located marketing module are also exploited by the SMARTBUY ecosystem's recommendation engine (see Sect. 5.1.4) in order to deliver relevant (i.e. user location history-informed) product recommendations to customers.

\subsubsection{Search and information retrieval}

This core module enables end-users (customers) to perform searches for products and services offered in nearby retailer stores ( $l$-commerce) over a unified, centralized inventory of products administered by the geographically distributed retailer-members of the SMARTBUY community. The module combines the search results with personalized product promotions and recommendations.

The module is accessed via the SMARTBUY native mobile application (see Fig. 5), which offers the following key functionalities (SMARTBUY 2019a; b):

- Location-based search for products and services, returning the list of products and stores which fulfill the search criteria.

- Application of several filtering options (maximum distance of retailers' location from the user's current location, product categories, price range) on location-based searches.

- Browsing of product details (title and description, attributes like price, size and color, availability) and store details (location, contact information, etc.).

- Adding products to a 'wishlist' or 'sharing' them with others.

- Reserving a product, to ensure its availability, for a limited period of time.

- Viewing promotional offers managed by the retailers as well as personalized lists of recommended products which are relevant to the customers' consumer profile and mobility pattern (see Sect. 5.1.4).

\subsubsection{Recommendation engine}

The employment of mobile recommendation engines (RE) in retailing serve as $\mathrm{m}$-shopping assistant systems which aim at providing consumers with meaningful recommendations 

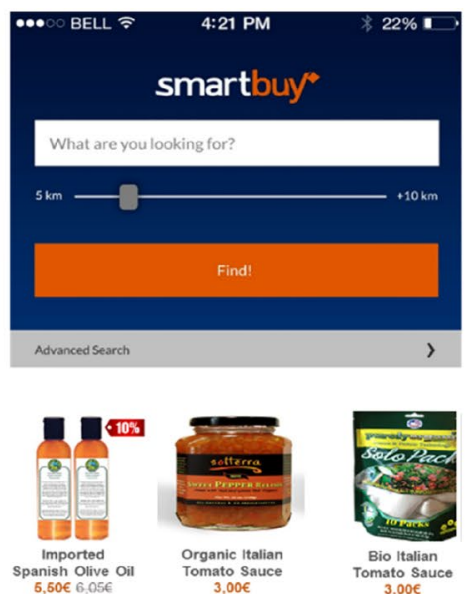

FEATURED PROMOTIONS
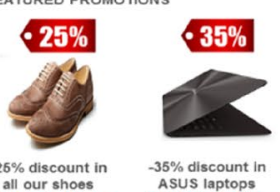

all our shoos

all our shoes
Visit Joe's Shoes

$-35 \%$ discount in
ASus laptops Asus laptops
Visit Micron Co.

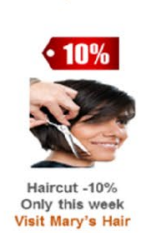

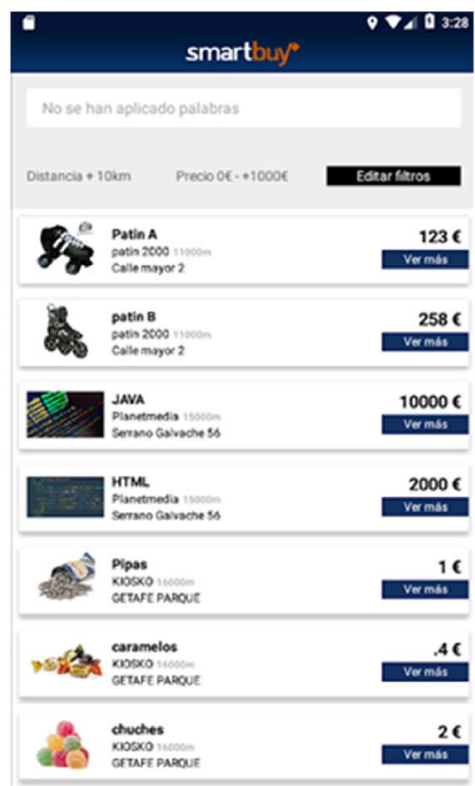

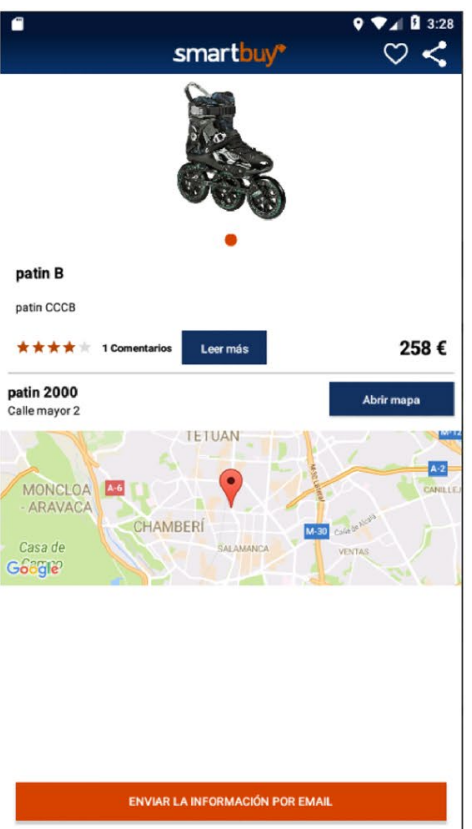

Fig. 5 Representative screens of the SMARTBUY app

(Walter et al. 2012). The recommendation lists may include products, services, offers, retailer shops, vendor-web sites, etc. The REs use algorithms that can either consider consumer data such as current position, shopping list, historical shopping, and browsing behavior (e.g. use keywords for product search or web site views) or refer to the consumer preferences on promotion offers, advertisements, and product attributes (e.g. similarity, proximity, price, and reputation) (Groß 2015).

The purpose of the SMARTBUY RE is to derive meaningful product recommendations addressed to mobile SMARTBUY users, so as to draw the attention of users to products they would potentially be interested in, but they would not discover themselves alone. The core RE algorithm of SMARTBUY employs a collaborative filtering $(\mathrm{CF})^{9}$ approach (Breese et al. 1998). The main research contribution of our work lies in that, among other contextual factors (like current location, time, season, consumer behavior), the SMARTBUY's RE also takes into account the location history of the target user for delivering more meaningful recommendations. In particular, location history is exploited in two ways: (a) to attain good matchings among pairs of users (intuitively, users who move around the same

\footnotetext{
${ }^{9} \mathrm{CF}$ is a method of making automatic predictions (filtering) about the interests of a user by collecting preferences or 'taste' information from many users (collaborating). The underlying assumption of the $\mathrm{CF}$ approach is that, if a person $\mathrm{A}$ has the same opinion as a person $\mathrm{B}$ on a subject, A is more likely to share B's opinion on a different subject than that of a randomly chosen person.
}

neighborhoods or visit shops of the same category will have interests or restrictions in common); (b) to recommended products sold on retailer stores relatively far from the user's current location, provided that the stores are located in areas frequently visited by the user. Moreover, interfacing with social media (Facebook) has been implemented in order to access basic user demographic information and be able to derive relatively accurate recommendations even when user ratings data are relatively sparse (Chatzidimitris et al. 2020; SMARTBUY 2019a, b).

The SMARTBUY mobile applications record in-app events (that is, events generated when users interact with the application, like keywords typed for product searches, product views, etc.). Effectively, the events are 'translated' to product ratings (e.g., when a user adds a product to her wishlist, this event is treated as a positive rating). The inapp events are timestamped (to capture the time context) and geo-stamped (to capture the mobility patterns of users, essentially, the GPS locations where they use the SMARTBUY app). Those data are uploaded, upon network availability, to the SMARTBUY's backend ecosystem (a web service endpoint has been implemented for that purpose) and stored in a database. SMARTBUY also makes use of WiFi APs deployed in the premises of retailers to accurately track user trajectories and record the number of users' 'physical' visits to stores (as explained in Sect. 5.1.2).

User profile data are also retrieved via the Facebook API (for those users who login though their Facebook account) and consolidated with the in-app activity data. In our CFbased approach, the RE identifies users whose in-app actions 
Fig. 6 The SMARTBUY's RE workflow diagram

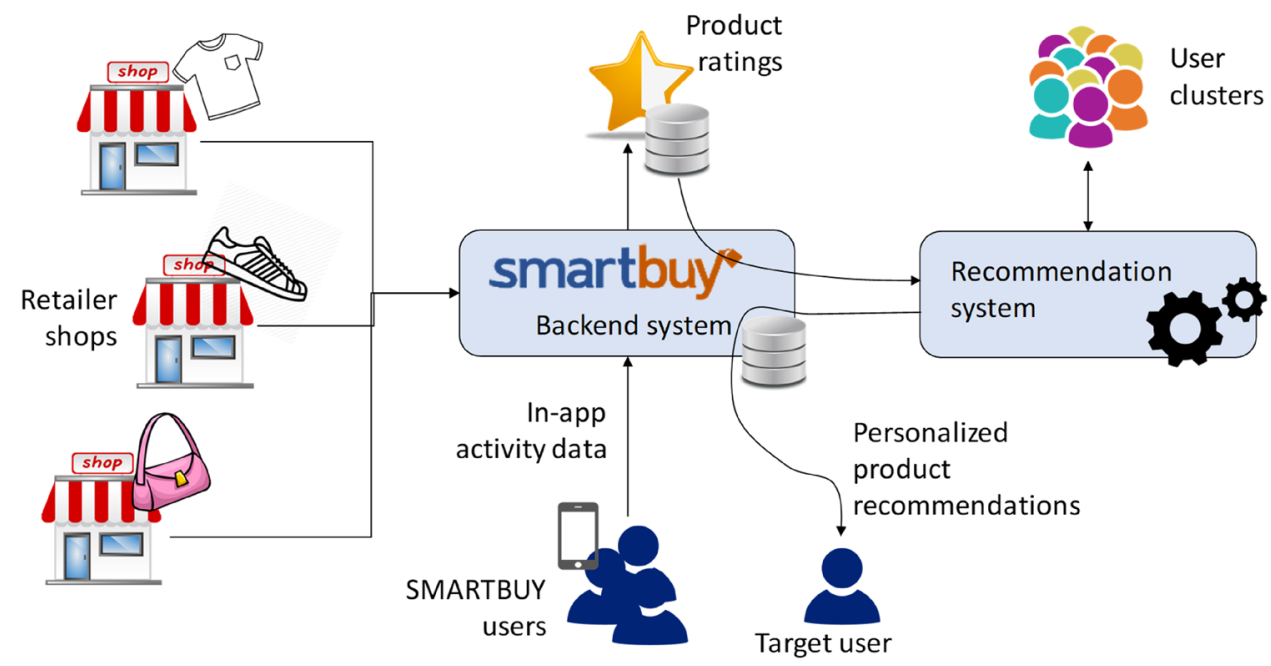

are similar to those of the target user and recommend products favored by like-minded users (which the active user has not yet considered). However, this approach does not consider items that no user has yet rated (this problem is known as the 'cold-start problem'). To address this issue, demographic data are used to cluster a user into an existing group of active users, even when no user in-app activity currently exists.

CF-based product recommendations adopt a user model wherein detailed information is collected about users and their preferences. The data is analyzed in order to determine like-minded users, namely users with similar preferences. The main objective is to create user groups (formally known as clusters) so that the group members share similar preferences. Then, when encountering a user $u$ interested in an item $i$, where $u$ belongs to a cluster of users with similar preferences, the algorithm may recommend $u$ products liked (i.e. highly rated) by other cluster members. The workflow diagram of the SMARTBUY's CF-based RE is illustrated in Fig. 6.

It is noted that the user-generated content (in-app activity data) are processed offline in order to form the clusters of users with similar preferences. This is a computationally intensive task, which is typically performed once per day. The output of this computation is the recommendation list of products per user so that the list is instantly delivered to any new (target) mobile user as soon as s/he logs into the ecosystem.

\subsection{Co-creation of iterative prototypes}

The creation of appealing digital products largely associates with the design of satisfactory and user-friendly experiences, which can favor market exploitation and customer retention. Towards this end, we design a user-driven solution that relates to the users' beliefs and perception and addresses their requirements. Our design is based upon an explicit understanding of users, the feedback actions and personalization processes and the corresponding living and working environments. The SMARTBUY platform has been implemented based on an innovative co-creation process consisting on a consultation of expectations from a group of stakeholders. Since the official launching of the project, we engaged with potential retailers and end-users (through focus group meetings, (in)formal interviews, informal meetings, etc.), thus facilitating the identification of the crucial requirements for the SMARTBUY platform.

In order to reach out to a broader community of retailers and users, we decided to use the large-scale experimentation (Amaxilatis et al. 2018) and co-creation (Gutiérrez et al. 2018) tools of the Organicity IoT platform and exploit the offered facilities to evaluate SMARTBUY in real-world conditions. We integrated the SMARTBUY services with the Organicity platform to set up an environment for conducting large-scale experimentations in a smart city with the SMARTBUY services. The results reported in Sect. 6 in relation to the pilots conducted in three European cities build upon the Organicity platform.

\subsubsection{The Organicity platform}

Organicity is a smart city platform that facilitates the involvement of different stakeholders (citizens, communities, scientists, developers, and others), and is not solely driven by engineering visions. It allows developers and researchers to create their own applications rapidly and easily using the provided datasets and tools to experiment with new ideas and innovative solutions. In this respect, Organicity provides a holistic citizen-driven approach for optimizing the city by combining the physical space with the digital.

Organicity's (OC) high-level architecture is illustrated in Fig. 7. It consists of three tiers: the OC Experimentation tier 


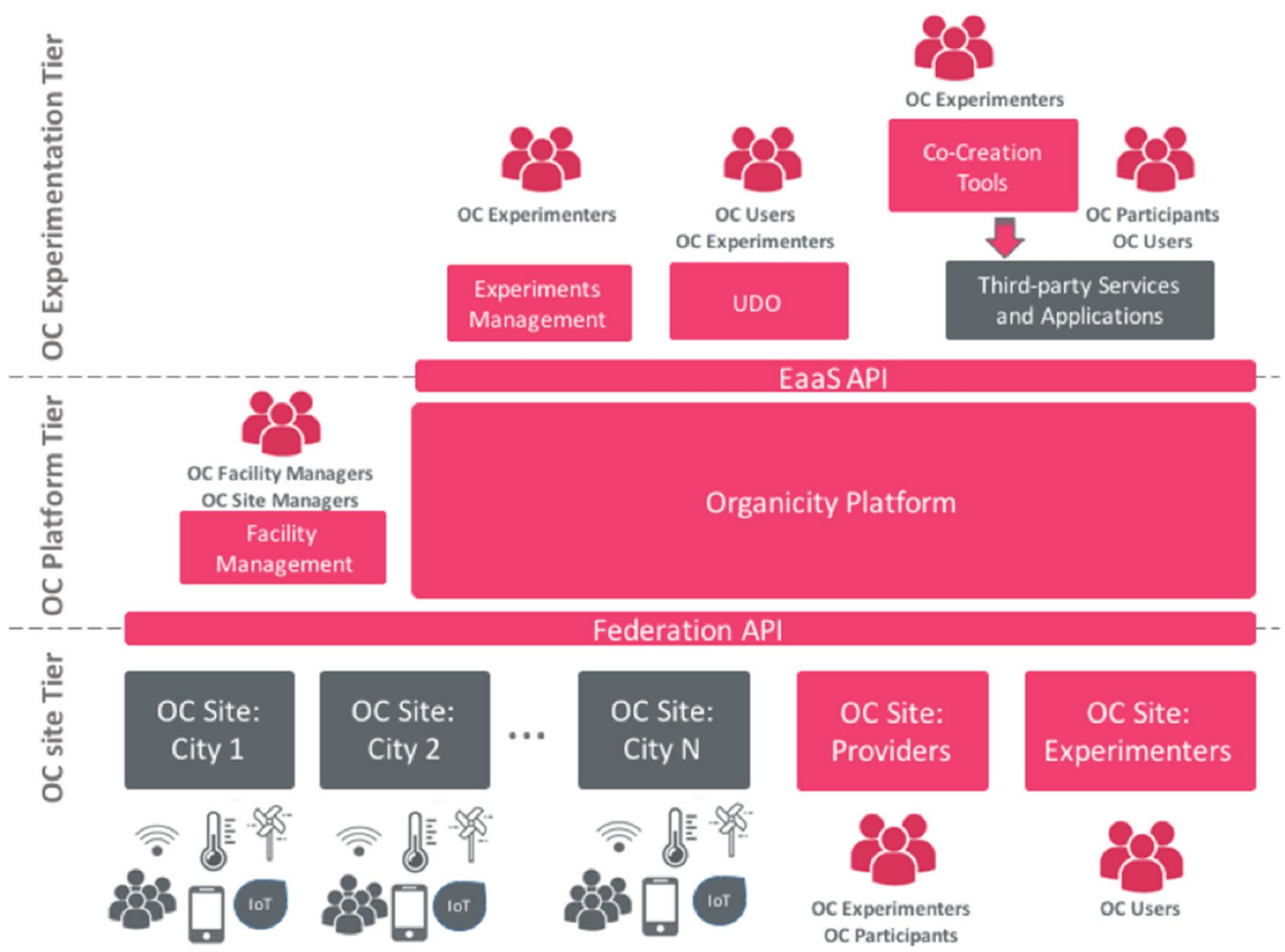

Fig. 7 The high-level architecture of Organicity

involves various components (deployed services and applications, template applications or libraries) that facilitate the building of applications and services for experimentation with them; the OC platform tier enables managers to administer and control the platform; the OC Site tier consists of different OC Sites (e.g., cities), the infrastructures and resources of experimenters (OC experimenters site) and the infrastructure supporting the feeding of crowdsourced data into the platform (OC Provider Site).

Organicity offers a built-in Experimentation-as-a-Service (EaaS) framework (see Fig. 7) which supports the co-creation and validation of a large set of smart city services and applications under real conditions in a powerful innovation ecosystem. It comprises a toolset that allows developing, deploying, and evaluating smart city solutions in a one-stop shop manner, allowing for large-scale experiments. One additional aspect of the EaaS framework is that it can be used to enable co-creation, i.e. to involve citizens and other stakeholders in designing as well as developing smart city solutions. The co-creation process with the different stakeholders and the different components that have been developed to create more inclusive innovation ecosystems, using the EaaS concept, is presented in (Gutiérrez et al. 2018).

The EaaS framework provides functionality to both advanced users/developers, as well as to more "casual" endusers like citizens, activists, decision makers or politicians, collectively referred to as Organicitizens. The idea is that any Organicitizen might develop and deploy her own services (e.g., a website, a web service, a desktop application or a smartphone application) that interact with the platform through the different APIs exposed by the Organicity facility.

Urban resources are modelled inside Organicity as Assets. These assets may model IoT sensor devices, observations (like temperature), information (like social events), smartphones, devices, services, data sources stored as data files (csv, xml, pdf) shared online, references to online data endpoints (third-party APIs and data services), places, buildings, and many other entities (including virtual objects).

An asset is associated with multiple attributes and each attribute can be associated with various metadata. Each Asset Attribute might represent various parameters of the asset. In the case of an IoT device or a smartphone, it might represent a sensing or actuation capability of the device or a device characteristic (static or not) like the human readable name of the device, its description, its location and so on. In the case of more abstract assets, attributes might represent types of observations not produced by physical sensors (e.g., a bus reporting the number of available seats or the number of the route). Each attribute consists of a type shared amongst all Organicity Sites, the actual value of the attribute and a list of metadata that describe the value. By utilizing the proper attributes for the description of Assets 


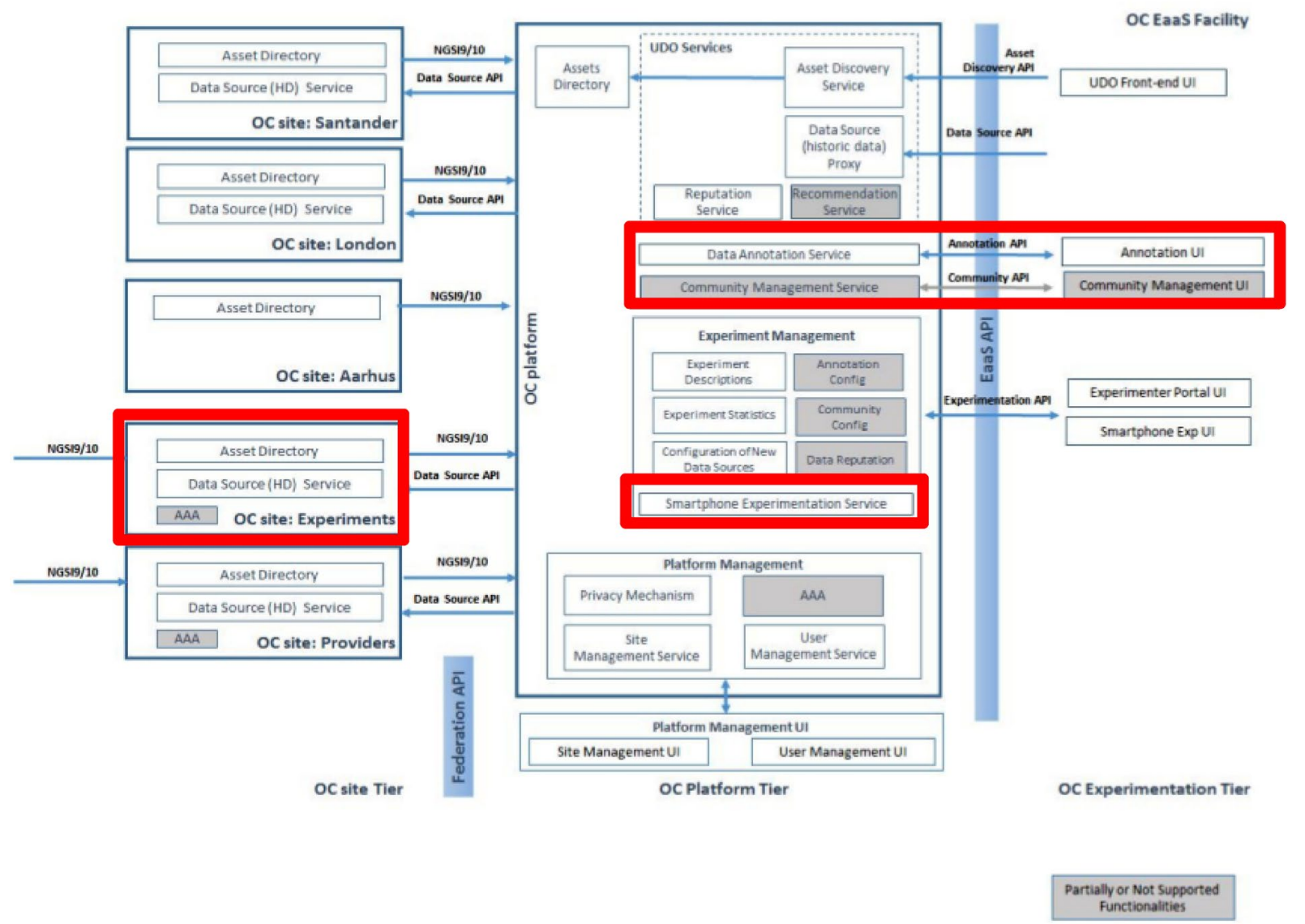

Fig. 8 The integration of SMARTBUY with Organicity. Adapted from Amaxilatis et al. (2018)

various visualization and browsing options are made available through the Urban Data Observatory (UDO) and the rest of the Organicity tools.

The Data Annotation Service manages the data annotation process on assets of all Organicity Sites. The primary goal of this service is to provide to the programmable clients an available set of tag domains and then receive and store tags (tag attachment on an asset by a user). The Organicity platform exposes the data annotation service through the Annotation API.

\subsubsection{Integration of SMARTBUY with Organicity}

The starting point of integration is to register SMARTBUY as an Organicity Site. We have implemented an Organicity Site Provider under the Organicity EaaS framework. As a result, all the data sets released by the SMARTBUY ecosystem (i.e., assets) can be accessed by the users of the Organicity facilities through the EaaS API and via the Urban Data Observatory website.

The following SMARTBUY data sets (assets) are released to the Organicity platform: the retailers data sets (providing information on the retailers enrolled in the
SMARTBUY ecosystem), the products and services data sets (providing information on all products and services registered within the SMARTBUY ecosystem by retailers), and the Wi-Fi location data sets (providing information on the Wi-Fi networks deployed through the retailers enrolled in the SMARTBUY ecosystem).

We also use the Annotation Service of Organicity to enrich the data sets with appropriate tags that can be exploited by any potential application that is envisaged to be developed.

The components in the Organicity architecture upon which the integration of the SMARTBUY ecosystem is materialized are illustrated in Fig. 8, enclosed in red boxes.

In the sequel, we shall briefly describe the set-up of an application that has been developed for the evaluation of retailers by customers (Amaxilatis and Giannakopoulou 2018), as a result of the aforementioned integration.

The specific application has been implemented in web and mobile app versions using the Organicity Experimentation as a Service framework and the associated tools provided. The application leverages a set of Organicity tools to provide a list of all available retailers around a 


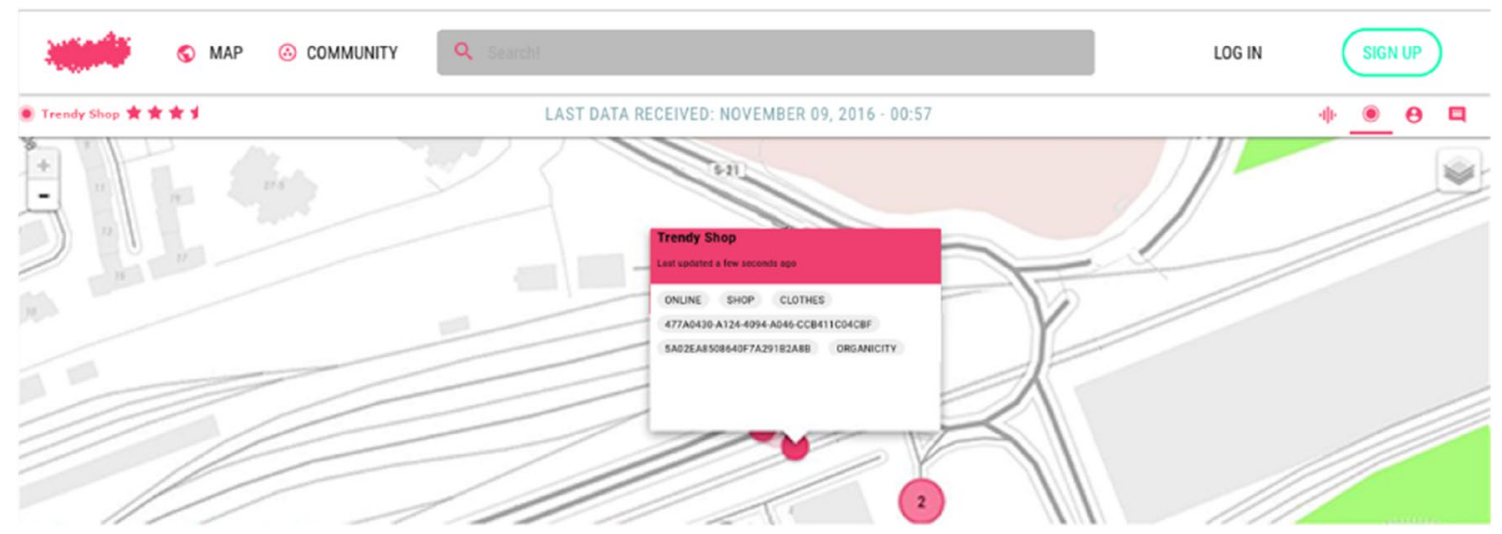

( $)$ ANNOTATIONS

How would you rate the politeness of this retailer

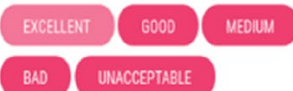

Your feectosck will help us generate move inowilogef for Patras and

improve our sutomated annotetion sentices.

(2) PROVIDER
Retailer Reputation: 3.5 / 5

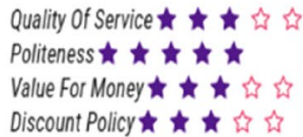

DiscountPolicy $t$

\section{Patras \\ - Patras, Greece}

Patras (or Patra) is Greece's third largest city and the regional capital of Western Greece, in northern Peloponnese, $215 \mathrm{~km}$ (134 mi) west of Athens. The city is built at the foothills of Mount Panachaikon, overlooking the Gulf of Patras.

Fig. 9 The Urban Data Observatory of SMARTBUY

customer, evaluate the retailers and assess the quality of service offered from each retailer.

The Asset Directory has been used to store and list all retailers who participated in the evaluation. The application has been part of an experiment set up in Organicity containing all the retailer's information including the name of the shop, the location, and a description of the shop's products and services. The Annotation Service has been used to enable customers to evaluate the retailers. This service was used to add a set of evaluation criteria per retailer as tags. Each tag provides a single aspect of a retailer among a list (e.g., quality of service, politeness, value for money, discount policy, etc.).

The actual evaluation could be carried out either through a smartphone or through the Urban Data ObservatoryUDO (web interface); cf. Fig. 9. UDO enables users to easily select tags defined for experiment assets (characterizing the retailer), e.g. to select the tag 'Excellent' for the asset 'Politeness'. Each customer can occasionally select multiple characterizations from the available ones and modify them at any time, thus affecting the overall evaluation of a retailer.

The outcome of the evaluation per retailer is the tag selected by the majority of customers for each of the tag domains defined. As a result of the rankings derived, customers are then able to search for products and services that match their requirements based on both the product specification and the retailer's characteristics. Retailers can have access to a dashboard system that presents them with information about their rankings and their overall evaluations fed by customers. Consequently, they could adapt and/or improve their behavior towards their customers and their store policies.

\section{Pilot study}

The pilots of the SMARTBUY ecosystem have been executed in 2018, in four European cities with diverse scale and retail market characteristics: London (UK), Patras (Greece), Luleå (Sweden) and Santander (Spain) (SMARTBUY 2019a; b).

As reported in Table 1, an overall number of 471 retailer stores joined SMARTBUY, created an account and uploaded products and promotions in the SMARTBUY ecosystem. A total of 2521 individuals installed the SMARTBUY app ${ }^{10}$ and acted as customers under real operational conditions.

\footnotetext{
$\overline{10}$ https://play.google.com/store/apps/details?id=com.smartbuysh opping.app.
} 
Table 1 Figures of customers/ retailers who actively participated in the pilot trials along with usage data logged during the trials

\begin{tabular}{llllll}
\hline & London & Patras & Luleå & Santander & Overall \\
\hline Retailers and customers & & & & & \\
Registered retailers & 235 & 110 & 40 & 86 & 471 \\
Retailers who filled in the questionnaire & 41 & 21 & 14 & 15 & 91 \\
User (customers) pilot participants & 1507 & 595 & 98 & 321 & 2521 \\
Customers who filled in the questionnaire & 113 & 105 & 24 & 98 & 340 \\
Products, promotions, products "near you" views, reservations & 1841 & 720 & 157 & 295 & 3013 \\
Products registered & 311 & 221 & 109 & 183 & 824 \\
Promotions & 14,090 & 9670 & 488 & 1221 & 25,469 \\
Product searches & 158 & 75 & 9 & 24 & 266 \\
Reservations & & & & & \\
\hline
\end{tabular}

\begin{tabular}{|l|c|}
\hline \multicolumn{1}{|c|}{ Types of Retailers visited } & Total Stores \\
\hline Clothes' shops (Men, Women, Kids) & 43 \\
\hline Accessories & 18 \\
\hline Aesthetic services (nails, etc) & 4 \\
\hline Hairdressing & 11 \\
\hline Shoes & 4 \\
\hline Opticians & 3 \\
\hline House equipment and decoration items & 12 \\
\hline Bookstores & 5 \\
\hline Pastry shops & 5 \\
\hline Gym & 3 \\
\hline Cellar & 2 \\
\hline TOTAL & $\mathbf{1 1 0}$ \\
\hline
\end{tabular}

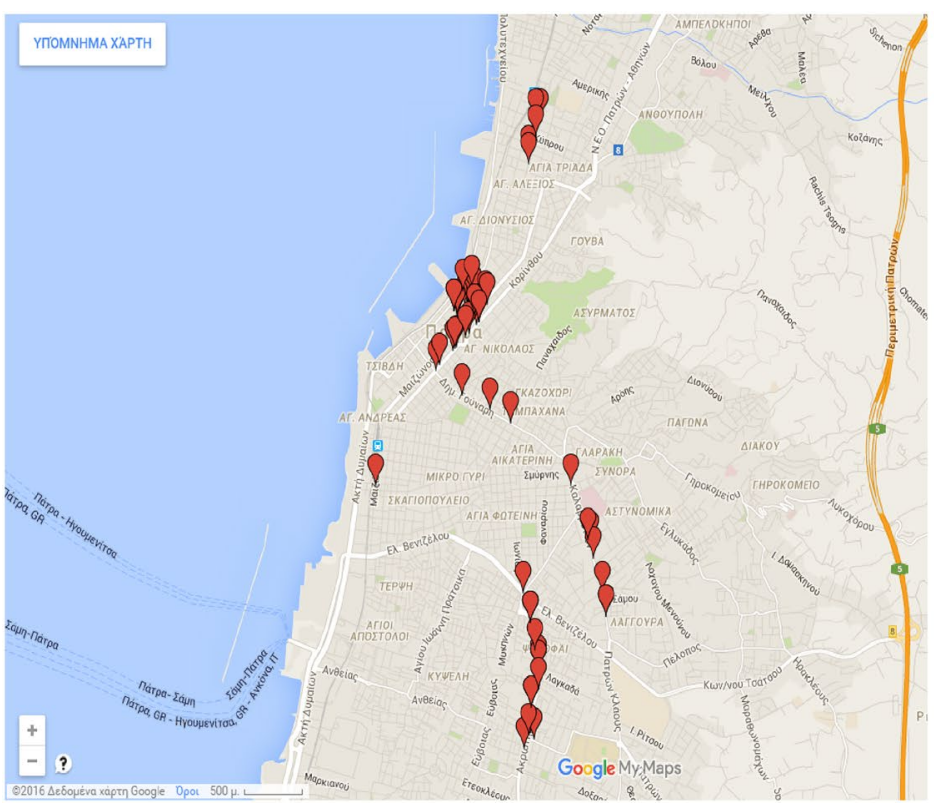

Fig. 10 Locations and categories of retailers' stores in Patras, Greece
Among them, 91 retailer shop owners (51\% females, $69 \%$ aged 35-54 years old) and 340 customers (51\% females, 53\% aged 19-34 years old), have accepted to evaluate their overall experience with the ecosystem through responding to an online questionnaire. It is noted that the questionnaire addressed to retailers has been distributed one month following the official launch of SMARTBUY in their area, while the customers' questionnaire has been embedded into the SMARTBUY app allowing users to easily submit their feedback.

As also shown in Table 1, the participating retailers have uploaded 6.4 products and 1.7 promotions on average through the retailer's web platform. Moreover, customers performed 10.1 product searches and 0.1 product reservations on average. According to the system logs, the customers commonly used the 'how to get there' feature to derive route directions towards selected retailer stores.
The data logged during the field trials have provided interesting insights with respect to the users' attitude towards SMARTBUY in different operational environments. For instance, retailers in small-to-medium sized cities (like Luleå) have mostly used the platform to deploy seasonal promotions rather than to advertise their offered products and services, probably because those are already known to their fellow citizens. Besides, the intensity of customers' activity (as dictated by location-based product searches) has been largely affected by the number of products available. Vice-versa, the engagement of retailers has been affected by the traffic generated by customers (i.e. interest for their promoted products). Interestingly, customers have been reluctant to reserve products, perhaps because the reservation period has been rather short (only a few hours), while reservations have been irrelevant for products with no stock restrictions (e.g. for takeaway coffee or hair cut services). 
Table 2 SMARTBUY evaluation from end-users (customers)

\begin{tabular}{|c|c|c|c|}
\hline QC\# & Questions (addressed to customers) & Avg & Median \\
\hline \multicolumn{4}{|c|}{ Generic questions } \\
\hline QC1 & I quite often buy products online & 3.7 & 4 \\
\hline QC2 & I prefer to collect the products I buy online from the physical store instead of having them shipped to me & 3 & 3 \\
\hline QC3 & When I shop online, I prefer to buy products from stores located in my area & 3.4 & 4 \\
\hline QC4 & Booking a product online and receiving it from the physical store is useful & 3.4 & 4 \\
\hline QC5 & Booking a product online and receiving it from the physical store helps me save shipping costs & 4.1 & 4 \\
\hline \multicolumn{4}{|c|}{ Usability } \\
\hline QC6 & The SMARTBUY application was fairly easy to use & 4.4 & 4.5 \\
\hline QC7 & The navigation within the SMARTBUY application was easy and comprehensible & 4.5 & 4.5 \\
\hline \multicolumn{4}{|l|}{ Utility } \\
\hline QC8 & By using the SMARTBUY application I discovered stores which I would not have discovered otherwise & 4.2 & 4 \\
\hline QC9 & $\begin{array}{l}\text { By using the SMARTBUY application I discovered products, sales and services which I would not have discovered } \\
\text { otherwise }\end{array}$ & 3.9 & 4 \\
\hline QC10 & Product recommendations have been relevant to my consumer profile and likings & 4.3 & 4 \\
\hline QC11 & Recommended products have been typically offered in stores conveniently located to city areas which I frequently visit & 4.4 & 4.5 \\
\hline
\end{tabular}

The locations and categories of retailer stores which joined SMARTBUY and participated in the pilot study in Patras are illustrated in Fig. 10.

Table 2 presents the eleven questions (QC) included in the questionnaire handed to 340 participants (customers) of the pilot phase. The table also illustrates a statistical compilation of the responses received (average and median values). The participants have been requested to reply using the Likert scale (1: strongly disagree, 5: strongly agree), while all questions allowed respondents to add free text in order to further elaborate on their answers.

Regarding the habits of customers who evaluated the SMARTBUY application, it appears that the majority buys products online rather often (QC1). Moreover, customers are somewhat divided as regards their preference on the means of delivery for products they have bought online between those that prefer to have them shipped to home and others that prefer to pick them up from the store to save waiting time (and cost) due to shipping (QC2). The preference to collect the purchased products from the store is mostly demonstrated in Patras and Santander, where the customers also tend to prefer buy products from local stores, even though they also practice online shopping (QC3). This finding is also verified by responses to QC4 and QC5 where customers in Patras and Santander expressed their appreciation for being able to collect a product bought online by the physical store, which also results in shipping cost savings. This aspect of SMARTBUY was also appreciated by customers in Luleå, yet, not in London where the longer travel times to reach the physical stores are deterring.

Responses to QC6 and QC7 reveal that the usability of the SMARTBUY application has been found acceptable in all cities. QC8 and QC9 also convey some interesting results. Many users in London, Patras and Santander argued that they discovered new stores, products, sales and services when using the SMARTBUY application, which they would have otherwise missed, revealing its usefulness in online promotion. That was not the case, though, in the smallerscale city of Luleå, where customers have been rather familiar with most stores.

The last two questions (QC10 and QC11) express the appreciation of customers as regards the performance of the recommendation engine; that is, the accuracy and relevance of recommended products both in terms of the actual type of products and the location of the stores where the products are sold.

A similar user survey has been conducted with retailers who joined SMARTBUY and participated in the pilots. The retailers have been first inquired regarding the marketing channels they use to promote their business. The vast majority among them actively advertise their products and services through online social networks (mostly Facebook and Instagram, 84\%) and Google Ads (59\%). Also, through business directories (57\%), dedicated website (33\%), local press and radio (29\%), flyers (26\%), advertising signs/magnets mounted on cars $(9 \%)$, etc. Table 3 presents the ten Likert-scale questions (QR) included in the questionnaire handed to 91 retailers.

The participating retailers have been satisfied with the usability aspects of the SMARTBUY ecosystem (QR1), however, not equally satisfied with the labor and time required to upload their products (QR2). In fact, some respondents argued that the ecosystem could support users to only enter a product's barcode and then automatically fill in its corresponding metadata. This finding explains why many users mostly used the ecosystem for uploading 
Table 3 SMARTBUY evaluation from retailers

\begin{tabular}{clcc}
\hline QR\# & Questions (addressed to retailers) & Avg & Median \\
\hline Usability & & 4.8 & 5 \\
QR1 & Using the SMARTBUY ecosystem was straightforward & 3.8 & 3.5 \\
QR2 & Uploading products and setting up promotions in SMARTBUY was fast and easy & 4.1 & 4 \\
Utility & & 4 \\
QR3 & I used the system mostly for uploading promotions rather than my whole inventory & 4 \\
QR4 & The SMARTBUY ecosystem is useful for promoting my products/services & 3.9 & 4 \\
QR5 & Using the SMARTBUY ecosystem allowed me to attract customers who I would not be able to reach otherwise & 3.3 & 4.5 \\
QR6 & I find that the SMARTBUY helps my business to compete large businesses, business chains and shopping malls & 4 \\
QR7 & I believe that my affiliation with SMARTBUY has had a positive impact on my overall business turnover & 3.6 & 4 \\
QR8 & I believe that my affiliation with SMARTBUY has had a positive impact with respect to non-economic factors & 4.1 & 4 \\
Attitude towards future involvement in the ecosystem & & 3.7 & 3.5 \\
QR9 & In the future, I would be positive in paying a reasonable fee to promote my products/services through SMARTBUY & 3.7 \\
QR10 & I would prefer if SMARTBUY offered the option for e-shopping & 3 \\
\hline
\end{tabular}

featured products or promotions rather than their whole inventory (QR3).

Overall, SMARTBUY has been valued as a useful instrument for promoting retailer businesses (QR4) and reaching new customers (QR5), especially in large city scales (like London). Most importantly, for supporting small retailers in competing business chains and shopping malls (QR6) through enabling business alliances and providing costeffective tools for personalized marketing. Many respondents have been somewhat uncertain with respect to the contribution of their affiliation with SMARTBUY on their overall business turnover, since the application only catered for reservation of products, yet, not for online purchases (QR7). However, most retailers found that SMARTBUY has been beneficial for their business for other reasons with no direct financial impact, like brand awareness (QR8). Thus, many retailers expressed their willingness to retain their SMARTBUY affiliation after the end the pilot period, at the expense of a reasonable subscription fee ( $Q R 9)$. Last, the potential future incorporation of e-shopping option in SMARTBUY (QR10) has been mostly appreciated by London-based retailers, however, many respondents have been skeptical due to the difficulty in competing the low product prices found in e-shop-only platforms.

\section{Conclusions and future work}

This article presents an overview of the SMARTBUY ecosystem, emphasizing on the motivation behind the research pursued as well as its main affordances and innovative aspects. SMARTBUY converts physical stores of smart cities into an open, geographically distributed mall by providing the logical consistency needed for conducting centralized searches over independent physical stores.
SMARTBUY is, firstly, innovative from a business perspective as it is the first known large-scale experimental ecosystem which implements a blended shopping paradigm: it combines the benefits of online shopping with the appeal of traditional store shopping. Moreover, it suggests a roadmap that achieves economies of scale for the, otherwise isolated, small retailers in urban environments, while also offering a suite of innovative services to customers. The core of the ecosystem is a module which enables customers to perform location-based searches for products and services offered by member-retailer stores, over a unified, centralized inventory of products. However, the key research contribution of the SMARTBUY ecosystem lies in two services: (a) the geo-location marketing service which takes advantage of a cloud-enabled city-scale cluster of WiFi APs to infer mobility patterns and consumer traits so as to push appropriate notifications to users; (b) a recommendation engine which considers a multitude of contextual factors like time, season, demographic data, consumer behavior, and location history of the user in order to accurately match like-minded customers and derive meaningful, personalized product recommendations.

The SMARTBUY ecosystem delivers several crucial benefits for retailers:

- Achieve economies of scale through enabling access to cloud-based inventory management and digital marketing services;

- Generate added value through becoming member of a broad market coalition and taking advantage of geomarketing opportunities;

- Enable broad visibility of offered products not only via the mobile app but also through supporting the indexability of the products uploaded in the retailers' web portal by web search engines; 
- Allow the effective targeting of potential customers through geo-marketing and intelligent context-aware recommendation systems, taking advantage of collected user profile information and user-generated data.

The SMARTBUY ecosystem also delivers important benefits to potential customers:

- Allow one-stop search over a large number of competitive shops in a similar fashion as practiced in brick-andmortar shopping malls or online shops;

- Enable feature and price comparisons, thus, achieving savings in shopping expenditures;

- Ensure awareness of -otherwise missed-promotional offers and would-like products, considering a multitude of contextual factors (some of which are not relevant to online customers), such as location \& location history, time, season, etc.;

- Combine the benefits of online shopping with those of traditional 'physical' shopping (social interaction, shopping entertainment, offline retailer loyalty, low product performance risk, prevention of shipment cost and delay, etc.).

The SMARTBUY ecosystem has undergone a large-scale pilot phase over a period of 12 months, in four European cities. The results have been overall positive. Most customers, especially those living in small-to-medium sized cities, appreciated the blended shopping model implemented in SMARTBUY due to allowing the comparison of prices online and the collection of purchased items from physical stores, thus saving cost and time associated with shipment. Many users also argued that they discovered new stores and products through using the SMARTBUY application. Last, most users assessed positively the recommendation engine, i.e., the accuracy and relevance of recommended products with respect to their likings and the context of use.

Moreover, the execution of the pilot trials provided valuable insights with respect to several technology acceptance factors which should be carefully considered when deploying smart retail alliances:

- The integration (or synchronization) with digital inventory systems is important to motivate retailers to upload a considerable percentage of their offered products or services; otherwise, systems similar to SMARTBUY are likely to be used solely for advertising promotions on selected products.

- The active involvement of a critical mass of both key actors, i.e. retailers and customers, is fundamental to ensure the long-term sustainability of the system: potentially high product availability intensifies customers engagement, while increased demand raised by custom- ers motivates retailers in uploading more products and promotions, thus producing a virtuous circle. Substantial resources should be invested in marketing campaigns to achieve this objective.

- The integration of payment gateways may enable online product purchases (similarly to e-commerce platforms) while still motivating users to visit the physical stores to pick up their bought products. Online payments could address both the reluctance of retailers to accept product reservations for long periods of time (since no guarantees are given for conversion of reservations to sales) and the requirement of customers to ensure the availability of reserved products.

Our future research will pursue the following objectives:

- Elaborate on suitable business models which will attract a critical mass of retailers and customers while also ensuring the financial sustainability of the SMARTBUY ecosystem.

- Design a coupon-like collaborative loyalty program (Villacé-Molinero et al. 2016), which will allow customers to collect and redeem coupons for product purchases from SMARTBUY retailer-members.

- Investigate solutions for brick-and-mortar stores, enabling them to tackle the challenges of health crises like the recent COVID-19 pandemic, e.g. scheduling the in-store pick-ups of online orders to enforce physical distancing and avoid queues, better employees' shift planning, adaptation of ventilation patterns based on predicted occupancy, etc.

Acknowledgements This research has been supported by the EU H2020 Programme under grant agreement no. 687960 (SMARTBUY). The research work of D. Gavalas and T. Chatzidimitris has been co-financed by the European Regional Development Fund of the European Union and Greek national funds through the Operational Program Competitiveness, Entrepreneurship and Innovation, under the call RESEARCH—CREATE-INNOVATE (project code: T1EDK-01572).

\section{References}

Amaxilatis et al (2018) Managing pervasive sensing campaigns via an experimentation-as-a-service platform for smart cities. Sensors 18(7):2125

Amaxilatis D, Giannakopoulou K (2018) Evaluating Retailers in a Smart-buying Environment using Smart City Infrastructures. In: 2018 International Conference on Pervasive Computing and Communications Workshops (PerCom). IEEE, pp 284-288

Amirkhanpour M, Vrontis D, Thrassou A (2014) Mobile marketing: a contemporary strategic perspective. Int J Technol Mark 9(3):252-269

Arora S, Singha K, Sahney S (2017) Understanding consumer's showrooming behaviour: Extending the theory of planned behaviour. Asia Pac J Mark Logist 29(2):409-431 
Bourg L, Chatzidimitris T, Chatzigiannakis I, Gavalas D, Giannakopoulou K, Kasapakis V, Zaroliagis C (2019) Enhanced Buying Experiences in Smart Cities: The SMARTBUY Approach. In: 2019 European Conference on Ambient Intelligence (AmI). Springer, pp 108-122

Bousios A, Gavalas D, Lambrinos L (2017) CityCare: crowdsourcing daily life issue reports in smart cities. In: 2017 IEEE Symposium on Computers and Communications (ISCC). IEEE, pp 266-271

Breese JS, Heckerman D, Kadie C (1998) Empirical Analysis of Predictive Algorithms for Collaborative Filtering. In: 1998 Conference on Uncertainty in Artificial Intelligence (UAI). AUAI, pp $43-52$.

Chatzidimitris T, Gavalas D, Kasapakis V, Konstantopoulos C, Kypriadis D, Pantziou G, Zaroliagis C (2020) A location history-aware recommender system for smart retail environments. Pers Ubiquit Comput 24(5):683-694

Chatzigiannakis I, Mylonas G, Vitaletti A (2011) Urban pervasive applications: challenges, scenarios and case studies. Comput Sci Rev 5(1):103-118

Chen BW, Ji W (2016) Intelligent marketing in smart cities: crowdsourced data for geo-conquesting. IT Prof 18(4):18-24

Gavalas D, Kenteris M (2011) A web-based pervasive recommendation system for mobile tourist guides. Pers Ubiquit Comput 15(7):759-770

Gavalas D, Kasapakis V, Konstantopoulos C, Mastakas K, Pantziou $\mathrm{G}$ (2013) A survey on mobile tourism recommender systems. In: 2013 Third international conference on communications and information technology (ICCIT). IEEE, pp 131-135

Gavalas D, Nicopolitidis P, Kameas A, Goumopoulos C, Bellavista P, Lambrinos L, Guo B (2017) Smart cities: recent trends, methodologies, and applications. Wirel Commun Mob Comput

Gensler S, Neslin SA, Verhoef PC (2017) The showrooming phenomenon: it's more than just about price. J Interact Mark 38:29-43

Giffinger R, Fertner C, Kramar H, Meijers E (2007) City-ranking of European medium-sized cities. Cent. Reg. Sci. Vienna UT 1-12

Gretzel U, Sigala M, Xiang Z, Koo C (2015) Smart tourism: foundations and developments. Electron Mark 25(3):179-188

Groß M (2015) Mobile shopping: a classification framework and literature review. Int J Retail Distrib Manag 43(3):221-241

Gutiérrez V, Amaxilatis D, Mylonas G, Muñoz L (2018) Empowering citizens toward the co-creation of sustainable cities. IEEE Internet of Things J 5(2):668-676

Hsiao MH (2009) Shopping mode choice: physical store shopping versus e-shopping. Transport Res Part E Logist Transport Rev 45(1):86-95

Kowatsch T, Maass W (2010) In-store consumer behavior: How mobile recommendation agents influence usage intentions, product purchases, and store preferences. Comput Hum Behav 26(4):697-704

Kumar TV, Dahiya B (2017) Smart economy in smart cities. Smart economy in smart cities. Springer, Berlin, pp 3-76

Lin C, Hong C (2008) Using customer knowledge in designing electronic catalog. Expert Syst Appl 34(1):119-127

Mehra A, Kumar S, Raju JS (2017) Competitive strategies for brick-and-mortar stores to counter "showrooming." Manag Sci 64(7):3076-3090
Meneses F, Moreira A (2012) Large scale movement analysis from WiFi based location data. In: 2012 International Conference on Indoor Positioning and Indoor Navigation (IPIN), pp 1-9

Mokhtarian PL (2004) A conceptual analysis of the transportation impacts of B2C e-commerce. Transportation 31(3):257-284

Pantano E, Priporas CV (2016) The effect of mobile retailing on consumers' purchasing experiences: a dynamic perspective. Comput Hum Behav 61:548-555

Pantano E, Timmermans H (2014) What is smart for retailing? Procedia Environ Sci 22:101-107

Pantano E, Rese A, Baier D (2017) Enhancing the online decision-making process by using augmented reality: a two country comparison of youth markets. J Retail Consum Serv 38:81-95

SMARTBUY Deliverable 3.2 (2017a) Wireless Geo-located marketing tool

SMARTBUY Deliverable 4.4 (2017b) Integration of advanced tools for products digitalization and monitoring

SMARTBUY deliverable 2.8 (2019a) SMARTBUY system-final prototypes

SMARTBUY Deliverable 5.4 (2019b) Deliverable 5.4 Report on feedback from real-life customers and retailers

Sanyal P, Ghosh A (2017) Attractiveness of retail agglomeration based on product type: an experimental study. SSRN 2989281

Sapiezynski P, Stopczynski A, Gatej R, Lehmann S (2015) Tracking human mobility using WiFi signals. PLoS ONE 10(7):e0130824

Sassi IB, Mellouli S, Yahia SB (2017) Context-aware recommender systems in mobile environment: on the road of future research. Inform Syst 72:27-61

Teller C (2008) Shopping streets versus shopping malls-determinants of agglomeration format attractiveness from the consumers' point of view. Int Rev Retail Distrib Consum Res 18(4):381-403

Theodoridis E, Mylonas G, Chatzigiannakis I (2013) Developing an IoT smart city framework. In: 4th International Conference on Information, Intelligence, Systems and Applications (IISA). IEEE, pp 1-6

Villacé-Molinero T, Reinares-Lara P, Reinares-Lara E (2016) Multivendor loyalty programs: influencing customer behavioral loyalty? Front Psychol 7:204

Walter FE, Battiston S, Yildirim M, Schweitzer F (2012) Moving recommender systems from on-line commerce to retail stores. IseB 10(3):367-393

Yang WS, Cheng HC, Dia JB (2008) A location-aware recommender system for mobile shopping environments. Expert Syst Appl 34(1):437-445

Yuan ST, Tsao YW (2003) A Recommendation mechanism for contextualized mobile advertising. Expert Syst Appl 24(4):399-414

Publisher's Note Springer Nature remains neutral with regard to jurisdictional claims in published maps and institutional affiliations. 